\title{
The use of next-generation sequencing in clinical diagnosis of familial hypercholesterolemia
}

\author{
Jana Vandrovcova, PhD', Ellen R.A. Thomas, MRCP', Santosh S Atanur, MSc', \\ Penny J. Norsworthy, BSc', Clare Neuwirth, SRN, RN ${ }^{1,4}$, Yvonne Tan, RN, BA ${ }^{1,4}$, \\ Dalia Kasperaviciute, $\mathrm{PhD}^{3}$, Jennifer Biggs, BSc, MRes', Laurence Game, PhD', \\ Michael Mueller, $\mathrm{PhD}^{3}$, Anne K. Soutar, $\mathrm{PhD}^{1}$ and Timothy J. Aitman, FRCP, DPhil ${ }^{1,3}$
}

\begin{abstract}
Purpose: Familial hypercholesterolemia is a common Mendelian disorder associated with early-onset coronary heart disease that can be treated by cholesterol-lowering drugs. The majority of cases in the United Kingdom are currently without a molecular diagnosis, which is partly due to the cost and time associated with standard screening techniques. The main purpose of this study was to test the sensitivity and specificity of two next-generation sequencing protocols for genetic diagnosis of familial hypercholesterolemia.
\end{abstract}

Methods: Libraries were prepared for next-generation sequencing by two target enrichment protocols; one using the SureSelect Target Enrichment System and the other using the PCR-based Access Array platform.

Results: In the validation cohort, both protocols showed $100 \%$ specificity, whereas the sensitivity for short variant detection was $100 \%$ for the SureSelect Target Enrichment and 98\% for the Access Array protocol. Large deletions/duplications were only detected using the SureSelect Target Enrichment protocol. In the prospective cohort, the mutation detection rate using the Access Array was highest in patients with clinically definite familial hypercholesterolemia (67\%), followed by patients with possible familial hypercholesterolemia (26\%).

Conclusion: We have shown the potential of target enrichment methods combined with next-generation sequencing for molecular diagnosis of familial hypercholesterolemia. Adopting these assays for patients with suspected familial hypercholesterolemia could improve cost-effectiveness and increase the overall number of patients with a molecular diagnosis.

Genet Med advance online publication 16 May 2013

Key Words: familial hypercholesterolemia; $L D L R$; molecular diagnostic testing; next-generation sequencing; target enrichment
Familial hypercholesterolemia (FH, OMIM no. 143890) is a common autosomal dominant condition with a prevalence of 1 in 500. Patients with FH have raised serum cholesterol levels and increased arterial deposition of low-density lipoprotein (LDL) cholesterol, leading to premature coronary heart disease. Despite the fact that early-onset coronary heart disease can be prevented by cholesterol-lowering drugs such as statins, less than a quarter of $\mathrm{FH}$ patients have currently been identified in the United Kingdom. ${ }^{1}$ Several diagnostic criteria have been developed to identify individuals with FH, including the Dutch Lipid Clinic ${ }^{2}$ criteria and the MedPed ${ }^{3}$ criteria. In the United Kingdom, the clinical diagnosis of FH is based on the Simon Broome criteria of cholesterol levels, presence of tendon xanthomata, family history, and genetic testing. ${ }^{4}$ The UK National Institute for Health and Clinical Excellence guidelines recommend initial mutation screening of index cases fulfilling Simon Broome criteria followed by cascade screening in at least firstand second-degree relatives. ${ }^{5}$

Most cases of FH are caused by mutations in the LDLR gene that encodes the LDL receptor protein, which binds LDL particles at the hepatic cell membrane and internalizes them for processing and excretion. FH-causing mutations in $L D L R$ are found throughout the gene and include missense, truncating, and splice site mutations; small insertion/deletion mutations; and large insertions/deletions that can encompass multiple exons. Some mutations have been found in many unrelated individuals with $\mathrm{FH}$, whereas others are found rarely. ${ }^{6}$ Mutations in two other genes, PCSK9 and $A P O B$, can also cause the FH phenotype but in $<20 \%$ of cases. ${ }^{7}$ Rare autosomal-recessive hypercholesterolemia is caused by mutations in the LDLRAP1 gene. $^{8}$

Conventional DNA testing of FH disease-causing genes is mostly based on direct capillary sequencing, with multiplex ligation-dependent probe amplification (MLPA) used for the detection of large insertions or deletions. ${ }^{9}$ These molecular techniques are sensitive and specific, but because of the cost and time involved, they are impractical for screening large numbers of patients. To overcome some of these limitations, assays such as the Amplification Refractory Mutation System (Elucigene FH20; Tepnel Molecular Diagnostics, Abingdon, UK) or arraybased sequencing methods (LIPOchip; Progenika Biopharma, Derio, Spain) have been developed. These assays are tailored to

The first three authors contributed equally to this work.

${ }^{1}$ MRC Clinical Sciences Centre, Faculty of Medicine, Imperial College London, London, UK; ${ }^{2}$ National Heart and Lung Institute, Imperial College London, London, UK; ${ }^{3}$ Institute of Clinical Sciences, Imperial College London, London, UK; ${ }^{4}$ Imperial College Healthcare NHS Trust, London, UK. Correspondence: Timothy J. Aitman (t.aitman@imperial.ac.uk) 
specific mutations and populations and therefore do not detect less common mutations or any novel mutations. ${ }^{10,11}$

During the past few years, high-throughput next-generation sequencing (NGS)-based methods have become available for DNA analysis. They have not only proven successful in new disease gene identification, but following the availability of economic "benchtop" sequencers, they have become more easily applicable to targeted diagnostic sequencing. The combination of high-throughput and relatively small DNA target selection allows for many genes and samples to be processed simultaneously, making it an attractive solution for the processing of large sample numbers in a diagnostic laboratory. Moreover, the flexible design of these assays enables molecular screening to be extended to other relevant genes and polymorphisms that are not covered by routine tests.

In this study, two target enrichment protocols, the hybridization-based SureSelect Target Enrichment System and the PCR-based Access Array System platform, followed by NGS sequencing, have been validated for the detection of FH-causing mutations. We show excellent performance for both approaches and discuss their potential for clinical screening programs and discovery of novel FH-causing genes.

\section{MATERIALS AND METHODS}

\section{Patients}

We studied two groups of patients, a validation group including patients with known molecular diagnosis and a prospective cohort of previously unscreened $\mathrm{FH}$ patients. In the validation group, DNA from 104 patients from the Hammersmith Hospital Lipid Clinic were analyzed. All 104 samples had previously undergone complete or partial screening of $L D L R$-coding regions and exon/intron boundaries, p.Asp374Tyr in PCSK9, and p.Arg3527Gln in $A P O B$ exon 26 using Sanger sequencing. In addition, MLPA was used to detect large deletions and duplications in a subset $(>80 \%)$ of the patients in the validation cohort, as described in the study by Tosi et al. ${ }^{9}$ Forty-five samples were known to carry heterozygous $\mathrm{FH}$-causing point mutations or small insertions/deletions $(<20 \mathrm{bp})$ in $L D L R$, PCSK9, or $A P O B$, and six had large heterozygous insertions/ deletions in $L D L R$. One sample was homozygous for $L D L R$ p.Gln384Pro, and one sample was a compound heterozygote with one missense mutation and one large deletion in $L D L R$. The remaining 51 samples in the validation cohort had negative molecular diagnoses. Of the 104 samples, 29 were processed using the SureSelect Target Enrichment System alone, 42 using the Access Array System microfluidic platform alone, and the remaining 33 using both platforms. In the prospective cohort, 84 consecutive patients referred for molecular testing by the Hammersmith Hospital Lipid Clinic over a period of 1 year were studied by the PCR-based Access Array System microfluidic platform. Fifteen of the 84 samples were also analyzed using the SureSelect Target Enrichment System. One individual was referred with suspected homozygous FH. All the remaining 83 were suspected to be heterozygous. Six patients had a diagnosis of definite FH based on Simon Broome criteria. The median highest total cholesterol in this group was $9.7 \mathrm{mmol} / \mathrm{l}$ (minimum: $7.9 \mathrm{mmol} / \mathrm{l}$; maximum: $15.9 \mathrm{mmol} / \mathrm{l})$. Sixty-five individuals had a diagnosis of possible $\mathrm{FH}$, with a median highest total cholesterol level of $8.7 \mathrm{mmol} / \mathrm{l}$ (minimum: $5.2 \mathrm{mmol} / \mathrm{l}$; maximum: $13 \mathrm{mmol} / \mathrm{l}$ ), and 13 patients did not fulfill Simon Broome criteria. The median highest total cholesterol in this group was $8.3 \mathrm{mmol} / \mathrm{l}$ (minimum: $6.3 \mathrm{mmol} / \mathrm{l}$; maximum: 9.6 $\mathrm{mmol} / \mathrm{l}$ ). The mean age at measurement was 43 years (minimum: 2 years; maximum: 68 years).

The study was approved under ethics committee references REC2002/6451 and REC11/LO/0883. All patients provided informed consent.

\section{DNA extraction}

DNA was extracted from blood using a standard phenol-chloroform protocol or the Maxwell 16 system (Promega, Madison, WI) or from saliva samples using the Oragene (Genotek, Ottawa, Ontario, Canada) protocol. Both protocols followed the manufacturer's recommendations.

\section{RNA extraction and reverse-transcription PCR}

Blood for RNA extraction was collected in Tempus blood RNA tubes (Applied Biosystems, Foster City, CA) and extracted using the Paxgene blood RNA kit (Qiagen, Hilden, Germany) following the manufacturer's protocol. Reverse transcription was performed using the iScript cDNA synthesis kit (BioRad, Hercules, CA). Primers (forward: TCGAGTTCCACTGCCTAAGTG and reverse: GTTGTTGTCCAAGCATTCGTT) were designed to amplify exons $4-7$ of $L D L R$.

\section{Custom SureSelect Target Enrichment System}

Four genes with mutations known to cause FH ( $L D L R, A P O B$, PCSK9, and LDLRAP1), a myopathy-associated variant in SLCO1B1, and 13 genes (APOE, HMGCR, HNRNPD, INSIG1, KHSRP, NPC1L1, PTBP1, SREBF1, SREBF2, MESDC2, SCAP, INSIG2, and CYP7A1) functioning within cholesterol-processing pathways were included in the SureSelect Target Enrichment System design (Agilent, Santa Clara, CA). The design contained 120-mer baits spanning the entire nonrepetitive sequence of the selected genes, including exons and introns, $2 \mathrm{~kb}$ of upstream sequence (10 kb for the four known FH genes), and $1 \mathrm{~kb}$ of downstream sequence.

The genomic coordinates of the 18 targeted genes were determined using the March 2006 build (NCBI36/hg18) of the human genome in the Ensembl genome browser. ${ }^{12}$ The density of bait tiling was fivefold, and the baits were allowed to overlap into repeat regions by $30 \mathrm{bp}$. The total targeted DNA length was $399 \mathrm{~kb}$. All libraries were generated from sheared DNA (Covaris, Woburn, MA) with an average insert size of $200 \mathrm{bp}$ following the SureSelect Target Enrichment System XT protocol for Illumina multiplexed sequencing version 1.2 (Illumina, San Diego, CA). After dilution to $2 \mathrm{nmol} / \mathrm{l}$, up to 30 libraries were pooled and sequenced on one lane on the HiSeq2000 platform (Illumina) to generate $2 \times 100 \mathrm{bp}$ paired-end reads. 


\section{PCR-based Access Array System}

The design included 43 amplicons covering all exons of $L D L R$, with the majority of the coding sequence covered by more than one overlapping fragment. Amplicons were also designed to cover exons 2, 4, 7, and 9 of PCSK9, containing the most common gain-of-function pathogenic mutations; $A P O B$ (one amplicon covering the most common familial defective apolipoprotein B-100 mutation, p.Arg3527Gln); APOE (one amplicon covering the APOE E2 variant site, rs7412); and SLCO1B1 (one amplicon covering rs4149056, the myopathy-associated variant). The average amplicon length was $184 \mathrm{bp}$, with $57 \%$ GC content. Primer sequences are shown in Supplementary Table S1 online. Samples were processed using the Access Array System (Fluidigm, South San Francisco, CA) according to the manufacturer's 4-Primer amplicon tagging protocol generating paired-end libraries for Illumina sequencing. Purified pooled products of 47 barcoded samples and one negative control were sequenced in one run using the MiSeq sequencer (Illumina). One amplicon, a GC-rich (75\%) amplicon in APOE targeting rs7412, failed to amplify and was excluded from subsequent data analysis.

\section{Data analysis}

Sequences were mapped to the GRCh37/hg19 human reference sequence using Burrows Wheeler Aligner v0.6.1. ${ }^{13}$ PCR duplicate reads were removed from SureSelect Target Enrichment System data (Picard tools v1.35; http://picard.sourceforge.net). Sequence reads from both data sets were further processed, and variants were called using GATK v1.0.6 ${ }^{14}$ with hard filtering options. Variant annotation was carried out with Ensembl's Variant Effect Predictor tool ${ }^{12}$ and was based on the transcripts ENST00000558518 (LDLR), ENST00000233242 (APOB), and ENST00000302118 (PCSK9). The annotation included Sorting Intolerant From Tolerant, Condel, and PolyPhen. Conservation scores (Genomic Evolutionary Rate Profiling scores) were obtained from Ensembl ${ }^{12}$ version 68 . To identify potentially pathogenic mutations, all nonsynonymous, splice site, frameshift, and truncating mutations were examined and compared with an $\mathrm{FH}$ locus-specific database ${ }^{6,15}$ as a guide to interpretation of variant pathogenicity. Synonymous and intronic variants located outside exon/intron boundaries as well as single-nucleotide polymorphisms with minor allele frequency $>1 \%$ in the International HapMap Project ${ }^{16}$ or the 1000 Genomes Project ${ }^{17}$ were excluded from further analysis. All single-nucleotide variants and short insertions/deletions that were potentially disease causing were verified by conventional Sanger sequencing. Novel variants were followed up by segregation analysis wherever possible.

\section{Copy-number variant analysis}

Copy-number variant (CNV) analysis from NGS data was performed for the samples sequenced using targeted capture. A read depth-based method, ${ }^{18}$ as implemented in $\mathrm{R}$ package ExomeDepth, was used to identify deletions and duplications spanning at least one exon. Each sequencing batch of samples was processed separately to increase the quality of a reference set for each sample and therefore to maximize the power to detect CNVs. Read depth was assessed for each exon in the target region, and the ratio of expected and observed read count was obtained, as well as a Bayes factor for the CNV calls, as implemented in the ExomeDepth method.

As an independent method of CNV analysis, MLPA was performed using the kit LDLR-P062 (MRC-Holland, Amsterdam, The Netherlands) following the manufacturer's protocol. The novel exon 16 deletion was confirmed by PCR. The previously described large deletions and duplications are detailed in the study by Tosi et al. ${ }^{9}$

\section{Statistical analysis}

The sensitivity of an assay was defined as the percentage of pathogenic mutations correctly identified with respect to previous or new Sanger sequencing and MLPA. The specificity is defined as the percentage of mutation-negative samples correctly identified as negative with respect to previous or new Sanger sequencing and MLPA.

\section{Myopathy-associated variant in SLCO1B1}

Genotypes of the SLCO1B1 myopathy-associated variant rs4149056 were scored from the Access Array System microfluidic platform and SureSelect Targeted Enrichment System data in all patients, and any history of adverse effects was obtained by review of medical records. Side effects were defined biochemically (transaminase or creatine kinase levels more than three times the upper limit of the normal range) or symptomatically for myalgia and other side effects that coincided temporally with statin treatment. Tests for deviation from HardyWeinberg equilibrium and association tests were performed using the DeFinetti software (http://ihg.gsf.de/cgi-bin/hw/ hwa1.pl).

\section{Validation study}

\section{RESULTS}

Custom SureSelect Target Enrichment System. To validate the assay, DNA was analyzed from 62 previously screened individuals who either had a confirmed molecular diagnosis of FH $(n=28)$ or were mutation-negative $(n=34)$. An average of $330 \mathrm{~Gb}(191-381 \mathrm{~Gb})$ of sequence was obtained per sample, with an average coverage of $\times 827$. Overall, $64 \%$ of total mapped reads aligned within the target region, and 99.8 and $98.8 \%$ of nucleotides were covered at $\times 4$ and $\times 25$, respectively. The insufficiently covered regions are consistent among runs and were found mostly outside coding regions. Hybridization-based capture is known to target GC-rich regions poorly; however, all regions containing known FH-causing mutations were covered sufficiently (more than $\times 25$ ) for confident variant calling. The initial analysis of the sequencing results was carried out by a researcher blinded to the gene and mutation details for each sample. All 20 heterozygous and 1 homozygous short pathogenic mutations, including point mutations and insertions/ deletions of $<15 \mathrm{bp}$, were detected (Table 1 ). In addition, one 
Table 1 Mutations identified in the validation study

\begin{tabular}{|c|c|c|c|c|c|}
\hline ID & $\begin{array}{c}\text { Detected by } \\
\text { SureSelect }\end{array}$ & $\begin{array}{l}\text { Detected by } \\
\text { Access Array }\end{array}$ & Gene & CDNA & Protein \\
\hline 990 & ND & Yes & $A P O B$ & c. $10580 \mathrm{G}>\mathrm{A}$ & p.Arg3527Gln \\
\hline 747 & Yes & Yes & $A P O B$ & c. $10580 \mathrm{G}>\mathrm{A}$ & p.Arg3527Gln \\
\hline 1086 & ND & Yes & $A P O B$ & c. $10580 \mathrm{G}>\mathrm{A}$ & p.Arg3527Gln \\
\hline 667 & ND & Yes & PCSK9 & c. $385 \mathrm{G}>\mathrm{A}$ & p.Asp129Asn \\
\hline 735 & ND & Yes & $L D L R$ & c. $1618 \mathrm{G}>\mathrm{A}$ & p.Ala540Thr \\
\hline 604 & Yes & Yes & $L D L R$ & c. $1880 C>A$ & p.Ala627Asp \\
\hline 660 & ND & Yes & $L D L R$ & c. $1048 C>T$ & p.Arg350X \\
\hline 728 & Yes & Yes & $L D L R$ & c. $1048 C>T$ & p.Arg350X \\
\hline 631 & Yes & Yes & $L D L R$ & c. $2231 \mathrm{G}>\mathrm{A}$ & p.Arg744Gln \\
\hline 384 & Yes & Yes & $L D L R$ & c. $1217 \mathrm{G}>\mathrm{A}$ & p.ArgR406GIn \\
\hline 895 & ND & Yes & $L D L R$ & c. $1691 A>G$ & p.Asn564Ser \\
\hline 896 & ND & Yes & $L D L R$ & c. $1691 A>G$ & p.Asn564Ser \\
\hline 766 & Yes & Yes & $L D L R$ & c.2061dupC & p.Asn688Glnfs*29 \\
\hline 430 & Yes & Yes & $L D L R$ & c. $661 \mathrm{G}>\mathrm{A}$ & p.Asp221Asn \\
\hline $570^{a}$ & Yes & Yes & $L D L R$ & c. $681 C>G$ & p.Asp227Glu \\
\hline 862 & ND & Yes & $L D L R$ & c. $681 C>G$ & p.Asp227Glu \\
\hline 1095 & ND & Yes & $L D L R$ & c. $1444 \mathrm{G}>\mathrm{A}$ & p.Asp482Asn \\
\hline 438 & Yes & Yes & $L D L R$ & c. $1444 \mathrm{G}>\mathrm{C}$ & p.Asp482His \\
\hline 1031 & ND & Yes & $L D L R$ & c. $401 \mathrm{G}>\mathrm{A}$ & p.Cys134Tyr \\
\hline 714 & Yes & Yes & $L D L R$ & c. $939 C>A$ & p.Cys313X \\
\hline 1067 & ND & Yes & $L D L R$ & c. $1130 \mathrm{G}>\mathrm{A}$ & p.Cys377Tyr \\
\hline 1068 & ND & Yes & $L D L R$ & c. $1130 G>A$ & p.Cys377Tyr \\
\hline 819 & Yes & Yes & $L D L R$ & c. 2029T>C & p.Cys677Arg \\
\hline 818 & ND & Yes & $L D L R$ & c. $2043 C>A$ & p.Cys681X \\
\hline 450 & Yes & Yes & $L D L R$ & c. $2043 C>A$ & p.Cys681X \\
\hline 814 & Yes & Yes & $L D L R$ & c. $2043 C>A$ & p.Cys681X \\
\hline 415 & Yes & Yes & $L D L R$ & c. $[1151 \mathrm{~A}>\mathrm{C}] ;[1151 \mathrm{~A}>\mathrm{C}]$ & p.Gln384Pro \\
\hline 813 & ND & Yes & $L D L R$ & c.303delG & p.Glu101Aspfs*105 \\
\hline 996 & ND & Yes & $L D L R$ & c. $301 \mathrm{G}>\mathrm{A}$ & p.Glu101 Lys \\
\hline 771 & ND & Yes & $L D L R$ & c. $301 \mathrm{G}>\mathrm{A}$ & p.Glu101Lys \\
\hline 820 & ND & Yes & $L D L R$ & c. $420 \mathrm{G}>\mathrm{C}$ & p.Glu140Asp \\
\hline 770 & Yes & Yes & LDLR & c.654_656delTGG & p.Glu219del \\
\hline 586 & Yes & Yes & LDLR & c. $682 \mathrm{G}>\mathrm{T}$ & p.Glu228X \\
\hline 1071 & ND & Yes & LDLR & c. $1646 \mathrm{G}>\mathrm{A}$ & p.Gly549Asp \\
\hline 853 & Yes & Yes & $L D L R$ & c. $1436 \mathrm{~T}>\mathrm{C}$ & p.Leu479Pro \\
\hline 857 & Yes & No & LDLR & c.2184_2194delGCTAAAGGTCA & p.Lys $730 \mathrm{Hisfs} * 48$ \\
\hline 743 & ND & Yes & $L D L R$ & c. $1 \mathrm{~A}>\mathrm{T}$ & p.Met? \\
\hline 663 & Yes & Yes & $L D L R$ & c.1118_1121dupGTGG & p. Tyr375Trpfs*7 \\
\hline 764 & Yes & Yes & $L D L R$ & c.1118_1121dupGTGG & p.Tyr375Trpfs*7 \\
\hline 1102 & ND & Yes & $L D L R$ & c. $1285 \mathrm{G}>\mathrm{A}$ & p.Val429Met \\
\hline 1104 & ND & Yes & LDLR & c. $1285 \mathrm{G}>\mathrm{A}$ & p.Val429Met \\
\hline 666 & Yes & Yes & $L D L R$ & c. $1285 \mathrm{G}>\mathrm{A}$ & p.Val429Met \\
\hline 852 & ND & Yes & $L D L R$ & c. $1586+5 G>C$ & \\
\hline 1011 & ND & Yes & $L D L R$ & c. $1706-1 \mathrm{G}>\mathrm{T}$ & \\
\hline 1094 & ND & Yes & $L D L R$ & c. $1845+1 \mathrm{G}>\mathrm{A}$ & \\
\hline 721 & ND & Yes & $L D L R$ & C. $1845+11 C>G$ & \\
\hline 566 & Yes & Yes & $L D L R$ & c. $313+1 \mathrm{G}>\mathrm{A}$ & \\
\hline 556 & Yes; Yes & Yes; No & $L D L R$ & c.[266G>A]; [2312-?_2547+?del] & $\begin{array}{l}\text { p.Cys89Tyr; } \\
\text { Exons 16-17 deletion }\end{array}$ \\
\hline 683 & Yes & No & $L D L R$ & c.-187-?_67+?del & Promoter and exon 1 deletion \\
\hline $836^{a}$ & Yes & No & $L D L R$ & c.-187-?_67+?del & Promoter and exon 1 deletion \\
\hline 547 & Yes & No & $L D L R$ & c.-187-?_67+?del & Promoter and exon 1 deletion \\
\hline $662^{\mathrm{a}}$ & Yes & No & LDLR & c.2312-?_2389+?del & Exon 16 deletion \\
\hline 436 & Yes & No & $L D L R$ & c.695-?_817+?del & Exon 5 deletion \\
\hline 564 & Yes & No & $L D L R$ & c.68-?_2583+?del & Exons 2-18 deletion \\
\hline 565 & Yes & No & $L D L R$ & c.68-?_940+?del & Exons 2-6 deletion \\
\hline 712 & Yes & No & $L D L R$ & c.1187-?_2140+?dup & Exons 9-14 duplication \\
\hline
\end{tabular}

Boldface indicates novel variants that were not previously identified.

CDNA, complementary DNA; ND, not done; ?, exact breakpoint boundaries not known.

aNew diagnosis in samples that had previously undergone partial screening only. 
compound heterozygote and six large insertions/deletions in the $L D L R$ gene were detected, resulting in $100 \%$ sensitivity for this assay. No false positives were detected, and specificity for this assay was also $100 \%$. A further three mutations were identified in $L D L R$ in patients without a previous molecular diagnosis. One was a known single-nucleotide substitution, p.Asp227Glu, and two were large deletions (deletion of exon 16 and deletion of promoter/exon 1). In addition, a novel variant was identified in the $L D L R$ promoter (c. $-227 \mathrm{G}>\mathrm{T}$; Genomic Evolutionary Rate Profiling score: 3.29; located in the highly conserved footprint 1 site), and two rare variants were identified in $A P O B$ in patients without a previous molecular diagnosis. The two variants, p.Pro877Leu (rs12714097) and p.Asp2213del (rs72653087), have not been described previously in $\mathrm{FH}$ patients and are not reported in either the 1000 Genomes Project ${ }^{17}$ or the International HapMap Project ${ }^{16}$ databases. No other rare missense variants were detected in the known FH genes in individuals without a molecular diagnosis.

PCR-based Access Array System. DNA samples from 53 previously characterized patients including 40 with point mutations ( 39 heterozygotes and 1 homozygote), 6 with insertions/deletions (all heterozygotes), 6 with large deletions or duplications (all heterozygotes), and 1 compound heterozygote with 1 missense mutation and 1 large deletion in LDLR (Table 1) were amplified together with samples from 22 patients who had been previously screened as negative for mutations. The median coverage per sample was $\times 572$ (minimum: 461; maximum: 625). All amplicons except APOE (see Materials and Methods) amplified with a mean coverage of $\times 506$. The coverage for individual amplicons is listed in Supplementary Table S2 online. Overall, 90\% of bases were covered more than 25 -fold. $L D L R$ had a mean coverage of $\times 656$ and $>98 \%$ of bases were above $\times 25$. All single-nucleotide changes in the coding sequence and intron/exon boundaries were correctly identified (Table 1), including one mutation in PCSK9 exon 2 and one in $A P O B$, despite the lower sequencing coverage of these amplicons with 13 and 15 reads, respectively (the percentage of mutated alleles was 54 and 53\%). One variant was not detected. This $11 \mathrm{bp}$ deletion (p.Lys730Hisfs ${ }^{\star} 48$ ) was not present in any aligned reads because the deletion overlapped with a forward primer, which prevented amplification of the mutated allele. The sensitivity for short variant detection was $98 \%$ ( 47 of 48 ), and specificity was $100 \%$. Similar to the SureSelect Target Enrichment System results, one mutation, p.Asp227Glu, was identified in a sample that previously had no molecular diagnosis. No pathogenic mutations were found in the remaining samples that were negative on previous screening. Large deletions could not be detected with the PCR-based Access Array System because no reduction in coverage was observed within deleted regions. The overall sensitivity of this assay as compared with SureSelect Target Enrichment System was therefore $82 \%$ (47 of 57).

\section{Prospective cohort}

To test the feasibility of an NGS-based mutation screening in a clinical setting, a consecutive cohort of 84 unrelated patients referred for genetic screening over a single year was analyzed. Sixty-nine of these patients were screened using the Access Array System assay alone, and 15 were processed using both protocols. Sequencing and coverage metrics are provided in Supplementary Table S2 online. In total, 23 variants that have previously been reported as pathogenic in $\mathrm{FH}$ patients were identified in 22 individuals (Table 2), including 13 single-nucleotide changes, 4 variants predicted to affect splicing, and 6 short insertions/deletions. In addition, two novel variants (p.Ala521Thr and p.Asn316Lysfs ${ }^{\star 54}$ ) were identified that had not been previously reported (Table 2 ). There was a good correlation between the results obtained from the Access Array System assay and SureSelect Target Enrichment System, with five of six mutations concordant between the data sets. The discordant variant, p.Lys730Hisfs ${ }^{\star} 48$, is the same one that was not detected using the Access Array System protocol in the validation cohort. Despite the presence of the same variant, these patients in the validation and prospective cohort were not known to be related. No additional mutations were identified using MLPA.

Of the two previously unreported variants (Table 2), the first was a missense change, p.Ala521Thr. This variant is conserved (Genomic Evolutionary Rate Profiling score: 2.66), is predicted to be damaging (PolyPhen score: 0.67, Sorting Intolerant From Tolerant score: 0.01 , and Condel score: 0.643 ), and segregates with affected status in the family (Supplementary Figure S1a online). The second variant was a single-nucleotide deletion in exon 7 of $L D L R$, p.Asn316Lysfs ${ }^{\star} 54$. This frameshift mutation creates a premature stop codon and also segregates with hypercholesterolemia in this family (Supplementary Figure S1b online).

Two (c.817+1G>A and c.941-4G>A) of the four splice site variants identified (Table 2) were previously detected in $\mathrm{FH}$ patients, ${ }^{6}$ but their pathogenicity had not been experimentally validated before. An RNA sample was available for one of them (patient ID: 35 ) and reverse-transcriptase PCR in this patient showed that the c. $817+1 \mathrm{G}>\mathrm{A}$ variant disrupted correct splicing (data not shown). Alternative splice products were generated by partial intron retention that led to truncation of the protein, by exon skipping that caused the loss of exon 5 sequence, and by a predicted frameshift of the remaining protein. Of the remaining variants previously identified in FH patients, one missense variant, p.Val827Ile, was conservative, without a published proof of pathogenicity and therefore was considered to be of unknown significance. Two children of this index case were available for segregation analysis, and the results showed that an unaffected daughter (total cholesterol: $4.6 \mathrm{mmol} / \mathrm{l}$; age: 27 years) of the proband had inherited the p.Val827Ile variant.

\section{Mutation detection rates}

The prospective cohort included six patients with definite $\mathrm{FH}$ as defined by the Simon Broome criteria, 65 with possible $\mathrm{FH}$, and 
Table 2 Previously reported and novel variants identified in known disease-causing genes in the prospective cohort

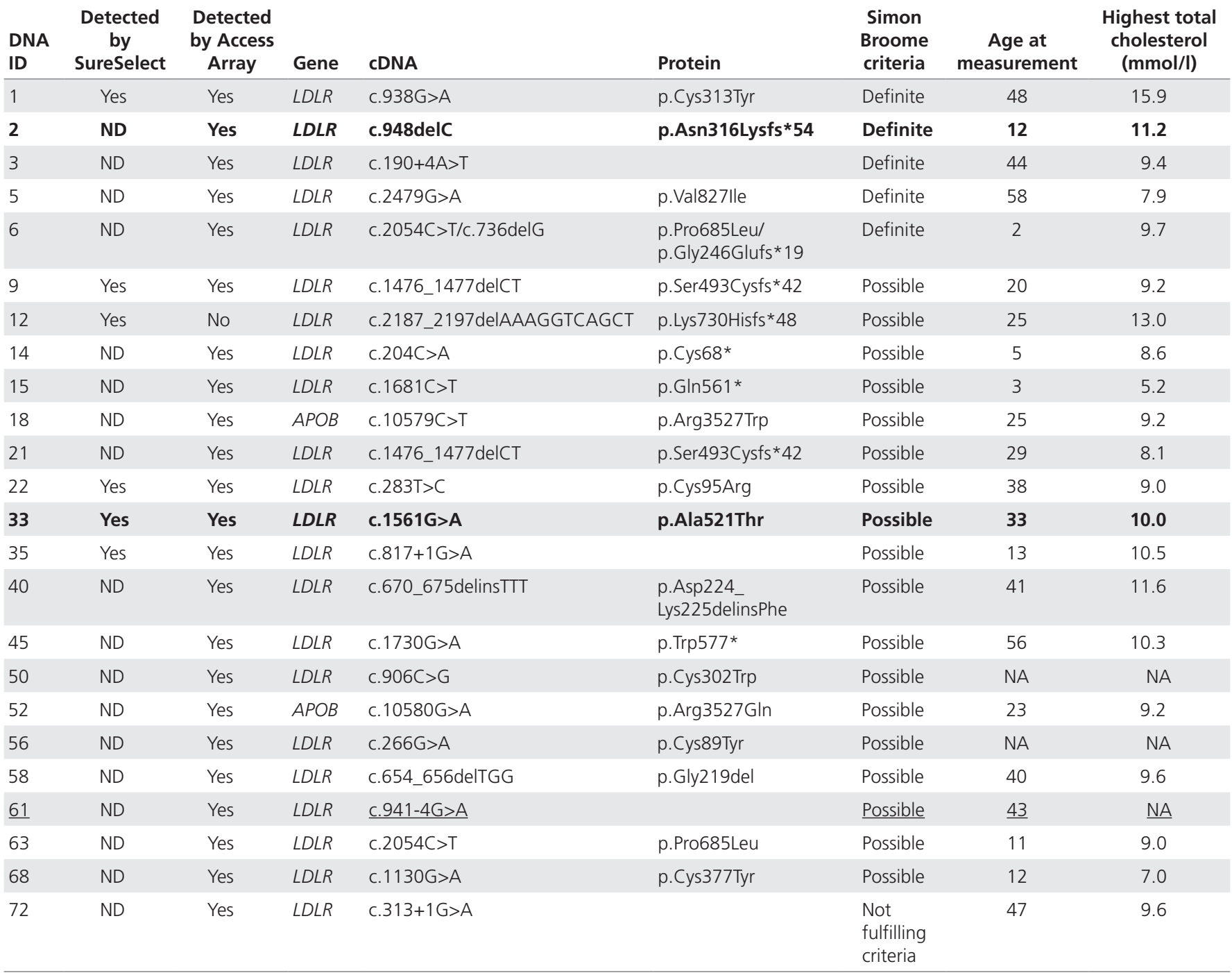

Boldface indicates novel, not previously identified variants. These variants are expected to cause and were shown to segregate with the disease. Underlining indicates variants of unknown significance. These rare variants were previously reported in patients, but their pathogenicity has not been established.

cDNA, complementary DNA; NA, not available; ND, not done.

13 hypercholesterolemic patients not fulfilling Simon Broome criteria for $\mathrm{FH}$. The highest detection rate of clearly pathogenic mutations was in the group of patients with a definite FH diagnosis (4 of $6,67 \%)$ followed by the group with a diagnosis of possible $\mathrm{FH}(17$ of $65,26 \%)$. One mutation (1 of $13,8 \%)$ was identified among the 13 hypercholesterolemic patients who did not fulfill FH diagnostic criteria.

\section{Variants in genes involved in cholesterol metabolism}

In addition to the three known FH-causing genes and SLCO1B1, the SureSelect Target Enrichment System assay included 13 genes that reside on cholesterol metabolism pathways. Rare variants identified in these genes, particularly in hypercholesterolemic individuals who had been screened negative for mutations in $L D L R, P C S K 9$, and $A P O B$, are potentially responsible for patients' raised cholesterol levels. Rare variants (minor allele frequency $<0.01$ ) identified in such individuals are listed in Supplementary Table S3 online. In patients with no previously known molecular diagnosis, seven rare nonsynonymous variants were found, of which six were not predicted to be functionally significant by Sorting Intolerant From Tolerant and PolyPhen. The single variant that was most likely to be of functional significance and therefore potentially pathogenic, was p.Val809Met in SREBF1. However, this was excluded from further analysis because it did not segregate with the phenotype in the family (data not shown).

\section{SLCO1B1 myopathy-associated variant}

Data on exposure to statins were available for 149 patients. Twenty-seven individuals were heterozygous for SLCO1B1 rs4149056, and two were homozygous. One patient (a heterozygote) had suffered severe biopsy-proven hepatitis while taking 
Table 3 The frequency of side effects of statin drugs in FH patients stratified by rs 4149056

\begin{tabular}{|c|c|c|c|c|}
\hline & Total & Heterozygous & Homozygous & Wild-type \\
\hline Creatine kinase $>3$ times the upper limit of normal range & 6 & 2 & 0 & 4 \\
\hline Creatine kinase $\leq 3$ times the upper limit of normal range & 5 & 1 & 1 & 3 \\
\hline Myalgia & 12 & 3 & 1 & 8 \\
\hline Transaminase levels $>3$ times the upper limit of normal range & 3 & 1 & 0 & 2 \\
\hline Transaminase levels $\leq 3$ times the upper limit of normal range & 9 & 2 & 0 & 7 \\
\hline Transaminase levels unknown & 3 & 1 & 0 & 2 \\
\hline Other patient-reported side effects & 11 & 4 & 0 & 7 \\
\hline Total & 49 & 14 & 2 & 33 \\
\hline
\end{tabular}

$\mathrm{FH}$, familial hypercholesterolemia.

statin drugs. A further 48 patients had suffered less-severe side effects, either patient-reported clinical effects or asymptomatic biochemical disturbances (Table 3). On association testing for rs4149056 in all individuals who had suffered side effects $(n=$ $49)$ versus those who had experienced no adverse effects on statins $(n=65)$, the odds ratio was 3.95 (confidence interval: $1.58-9.89 ; P=0.002)$.

\section{DISCUSSION}

We investigated the sensitivity and specificity of two target enrichment protocols, combined with NGS, for the detection of disease-causing mutations in patients with proven or suspected FH. Using the SureSelect Target Enrichment System and the HiSeq 2000 system, $98.8 \%$ of targeted regions were covered at more than $\times 25$ as compared with the PCR-based Access Array System followed by MiSeq sequencing, for which on average $10 \%$ of nucleotides failed to reach this coverage. Regions with low coverage were found outside the coding sequence of $L D L R$ and mutation hotspots in $A P O B$ and PCSK9 and therefore did not affect the overall success of mutation detection. Both techniques showed high sensitivity and specificity for the detection of single base substitutions and short insertions/deletions. In the validation part of the study, $100 \%$ of previously detected mutations were correctly identified using the SureSelect/HiSeq protocol. In comparison, the Access Array System/MiSeq approach led to correct identification of $98 \%$ of all variants detected previously by Sanger sequencing, although large insertions/deletions could not be detected (see below). The single variant that was not detected by the PCR-based Access Array System protocol was an $11 \mathrm{bp}$ deletion (p.Lys730Hisfs ${ }^{\star} 48$ ) that overlapped a primer site. However, the recent availability of longer sequencing reads, currently up to $250 \mathrm{bp}$ when using MiSeq, will be anticipated in most cases to eliminate the need to have primer sites within coding regions or allow design modification to include longer overlaps between amplicons.

Three mutations in the LDLR coding sequence (one singlenucleotide variant, p.Asp227Glu, and two large deletions) and one $L D L R$ promoter variant (c. $-227 \mathrm{G}>\mathrm{T}$ ) were identified in patients who were previously classified as negative for mutations. Inspection of the previous laboratory database indicated that the p.Asp227Glu variant and the two large deletions had not been detected because of incomplete capillary sequencing in the case of the p.Asp227Glu and promoter variants and absence of MLPA data in the case of the two deletions.

A small proportion of $\mathrm{FH}$ cases are caused by large deletions or duplications of $L D L R .^{19}$ The current standard screening is based on MLPA, a technique that is highly reliable but that is a costly and time-consuming addition to sequencing. The detection of large variants from NGS data has been shown previously, but its use in FH diagnostics has not yet been investigated. ${ }^{20,21}$ Here, the combination of SureSelect Target Enrichment System/ HiSeq and data analysis using ExomeDepth software ${ }^{18}$ led to correct identification of all eight large deletions and one large duplication. This shows the potential of using hybrid capture for the detection of both short and large sequence variants in FH. The read depth from the PCR-based Access Array System assay was not used for this analysis method because read depth in this study did not correlate with exon deletions, although it has recently been shown that amplicon multiplex PCR can be optimized for CNV detection..$^{22}$

When testing a new molecular workflow for routine diagnostics, in addition to sensitivity and specificity, it is important to consider the cost and time efficiency of the protocol. The SureSelect Target Enrichment System protocol allows for comprehensive coverage of targeted regions (current custom design up to $24 \mathrm{Mb}$ ), whereas the Access Array System protocol is limited to 480 amplicons with a maximum length of $400 \mathrm{bp}$ when sequenced using the latest MiSeq system. Sequence capture also allows the detection of all types of variants, including large deletions and duplications, which was not possible in this study using the PCR-based Access Array System. On the other hand, the Access Array System is considerably cheaper to run, ${ }^{20,23}$ with reagent costs approximately 10 -fold lower than those for the SureSelect Target Enrichment System protocol, ${ }^{23}$ and, in addition, the library preparation turnaround time is shorter. In our hands, 96 samples can be processed within a day using the Access Array System protocol as compared with at least 3 days needed for the SureSelect Target Enrichment System insolution capture. Initial DNA requirements of the Access Array System/MiSeq protocol are also low (50 ng vs. $1 \mathrm{ug}$ ), and most of the process is automated, reducing the risks of contamination and human error to a minimum. Recently, Hollants et al. ${ }^{24}$ 
published a protocol for $\mathrm{FH}$ mutation detection that was also based on the Access Array System, but the amplicons were sequenced using the pyrosequencing-based GS-FLX (Roche, Branford, CT) platform. They successfully identified all short variants but, as with our study, could not detect large rearrangements. Our Access Array System protocol is here combined with the MiSeq sequencing platform (a benchtop personal NGS system specifically developed to suit the needs of the routine laboratory setting) and offers similar quality to GS-FLX but at a lower sequencing cost. ${ }^{25}$ The use of the MiSeq system is also reported to limit errors of variant calls within homopolymer regions that are known to occur in pyrosequencing. ${ }^{26}$

\section{Novel variants identified in the validation study}

The $L D L R$ promoter variant c. $-227 \mathrm{G}>\mathrm{T}$ has not previously been reported in FH patients, or in other populations, but was investigated previously as part of a study delineating the conserved footprint 1 site. A luciferase assay showed that the c. $-227 \mathrm{G}>\mathrm{T}$ variant had $\sim 75 \%$ transcription levels as compared with the wild-type site. ${ }^{27}$ Although a $25 \%$ reduction is not a definitive decrease in promoter activity, it cannot be excluded that this change is sufficient to cause raised cholesterol levels in this patient. Family members were unavailable for segregation anal$y$ sis, and we therefore classify this variant as being of uncertain significance pending further functional and segregation data. The remaining two novel variants in $A P O B$, rs12714097 and rs72653087, were located outside $L D L R$-binding sites, regions currently not associated with hypercholesterolemia, and therefore their pathogenicity remains to be elucidated.

\section{Genotype-phenotype correlation in the prospective cohort} In the United Kingdom, FH diagnosis is made based on the Simon Broome criteria that identify patients with definite or possible FH. Following the National Institute for Health and Clinical Excellence guidelines, ${ }^{6}$ genetic testing is recommended for all suspected index cases and should be followed by family cascade screening if a mutation is found. Our study focused on a group of consecutive patients referred for genetic screening by a local lipid clinic over the course of 1 year.

To assess clinical significance, all rare variants (minor allele frequency $<0.01$ ) that were identified in $L D L R, A P O B$, or PCSK9 were compared against locus-specific FH databases. ${ }^{6,15}$ All nonsense variants and insertion/deletion variants that have been found previously among $\mathrm{FH}$ patients were also considered pathogenic. Missense variants were considered to be pathogenic if they were predicted by Sorting Intolerant From Tolerant and PolyPhen to be deleterious and probably damaging, respectively, and were previously identified in $\mathrm{FH}$ patients. On the basis of these criteria, a missense change, p.Val827Ile, was included among the list of pathogenic variants, although the conservative valine to isoleucine substitution may not affect protein function. This residue is the third amino acid within the internalization signal for LDLR, NPVY, a position generally not necessary for correct internalization. ${ }^{28}$ In addition, our segregation data suggest that this variant may not be disease causing, and we therefore classified this variant as being of unknown significance. Of the four splice site variants, three (c.313+1G $>A$, c. $190+4 \mathrm{~A}>\mathrm{T}$, and $\mathrm{c} .817+1 \mathrm{G}>\mathrm{A}$ ) were shown experimentally to disrupt splicing, two in published data ${ }^{29,30}$ and one within this study, and are therefore listed as pathogenic. The remaining splice variant, c.941-4G $>A$, was described previously in $\mathrm{FH}$ patients, but its pathogenicity remains to be experimentally confirmed. An RNA sample from this individual was not available for confirmation within this study.

The mutation detection rates among patients with definite (66\%) and possible (26\%) FH are comparable to those of previously published studies. ${ }^{7}$ One mutation was identified among 13 patients who did not fulfill Simon Broome criteria. This suggests that a number of patients with high cholesterol in the general population who do not fulfill formal clinical criteria for FH diagnosis may have FH-causing mutations. Such patients may therefore be diagnosed by the assays developed here, and, because these mutations are dominantly inherited, family cascade screening would probably identify the same mutations in $50 \%$ of their first-degree relatives.

The types of mutation identified here reflect the distribution of variants published in the $L D L R$ locus-specific database, ${ }^{15}$ with exonic substitutions (56\%) being the most common, followed by short insertions/deletions (28\%). Most of the mutations identified here were unique, with only $20 \%$ common among UK FH patients that would be identified using the Elucigene FH20 commercial ARMS kit. In our prospective study, we did not identify any mutations in PCSK9 or large rearrangements of $L D L R$. These variants are generally rare among patients with $\mathrm{FH},{ }^{7}$ and we therefore consider it unlikely that our results are biased in any way.

Recently, the importance of screening additional regions of $A P O B$ has been highlighted by Motazacker et al. ${ }^{31}$ who identified two patients with novel mutations located outside the commonly sequenced region of $A P O B$ exon 26 . Screening of entire coding regions instead of focusing on mutation hot spots is therefore likely to enhance the discovery of FH-causing mutations. The format of next-generation assays is flexible and can be readily extended to include full coding regions of $A P O B$ and PCSK9, as well as coding regions of other medically relevant genes, such as APOE and SLCO1B1, which currently need to be genotyped separately.

Our current Access Array System covers the same regions that would be screened using conventional Sanger sequencing, and therefore the number of variants identified would be no larger than that identified after Sanger sequencing. The SureSelect Target Enrichment System design also included genes in cholesterol metabolism pathways and regions of $A P O B$ and PCSK9 outside those known to be causative of $\mathrm{FH}$. To establish the pathogenicity of such variants, further in silico and experimental analyses will be required. For detection of variants outside known FH-causing genes or gene regions, we focused on variants that are rare (minor allele frequency $<0.01$ ), are probably pathogenic (i.e., located in coding regions, promoters, and exon/intron boundaries), and 
are present only in individuals without an existing molecular diagnosis of FH. Using these filters, we were left with a manageable list of variants $(<10)$ that we decided to follow up. When screening larger regions in more patients, the identification of large numbers of variants of unknown significance will necessitate further extensive family segregation and functional assays. However, the increase of data available in public databases and improvements of bioinformatics tools will allow more efficient filtering of variants than is available at present.

\section{Statin-induced myopathy}

Previous published data on larger cohorts show an odds ratio of 4.5 for association of rs 4149056 with myopathy, ${ }^{32}$ and on that basis, guidelines have recommended avoiding higher doses of simvastatin in heterozygotes or homozygotes for rs4149056. ${ }^{33}$ Association testing restricted to more severe side effects (transaminases or creatine kinase greater than three times the upper limit of normal) was not informative in our cohort due to the small sample size. However, less-severe patient-reported adverse effects to statin treatment such as myalgia have an effect on patient adherence to treatment in this high-risk population. Therefore, the association identified here between SLCO1B1 genotype and a wider range of milder adverse effects in this lipid clinic cohort gives an insight into the potential benefits of prospective genotyping before the initiation of statin treatment.

In conclusion, we have shown the potential utility of sequence target enrichment methods in combination with NGS in molecular diagnostics of FH. Owing to the comprehensive coverage, SureSelect Target Enrichment System protocols (either whole-exome or region-specific) may provide the most benefit for studies that aim to identify novel disease-causing genes and for diseases for which the number of genes needing to be screened is very large. In FH diagnostics for which $<5$ genes need to be analyzed, PCR-based enrichment techniques offer more streamlined protocols that provide high sensitivity for mutation detection and may offer an affordable option for clinical screening of large numbers of patients with suspected FH. If adopted, greater numbers of $\mathrm{FH}$ patients and their relatives may potentially benefit from early diagnosis and treatment.

\section{SUPPLEMENTARY MATERIAL}

Supplementary material is linked to the online version of the paper at http://www.nature.com/gim

\section{ACKNOWLEDGMENTS}

We acknowledge funding from the MRC Clinical Sciences Centre, from the British Heart Foundation to S.A. and T.J.A.; from a Wellcome Trust Clinical Training Fellowship to E.R.A.T.; and from the National Institute for Health Research-funded Imperial Biomedical Research Centre to D.K., M.M., and T.J.A.

\section{DISCLOSURE}

The authors declare no conflict of interest.

\section{REFERENCES}

1. Marks D, Thorogood M, Farrer JM, Humphries SE. Census of clinics providing specialist lipid services in the United Kingdom. J Public Health (Oxf) 2004;26:353-354.

2. Defesche JC. Familial hypercholesterolemia. In: Betteridge DJ (ed). Lipids and Vascular Disease. Martin Dunitz: London, 2000:65-76.

3. Williams RR, Hunt SC, Schumacher MC, et al. Diagnosing heterozygous familial hypercholesterolemia using new practical criteria validated by molecular genetics. Am J Cardiol 1993;72:171-176.

4. Scientific Steering Committee on behalf of the Simon Broome Register Group. Risk of fatal coronary heart disease in familial hypercholesterolaemia. BMJ 1991;303(6807):893-896

5. Wierzbicki AS, Humphries SE, Minhas R; Guideline Development Group. Familial hypercholesterolaemia: summary of NICE guidance. BMJ 2008;337: a1095.

6. Leigh SE, Foster AH, Whittall RA, Hubbart CS, Humphries SE. Update and analysis of the University College London low density lipoprotein receptor familial hypercholesterolemia database. Ann Hum Genet 2008;72(Pt 4):485498.

7. Taylor A, Wang D, Patel K, et al. Mutation detection rate and spectrum in familial hypercholesterolaemia patients in the UK pilot cascade project. Clin Genet 2010;77:572-580.

8. Soutar AK, Naoumova RP. Mechanisms of disease: genetic causes of familial hypercholesterolemia. Nat Clin Pract Cardiovasc Med 2007;4:214-225.

9. Tosi I, Toledo-Leiva P, Neuwirth C, Naoumova RP, Soutar AK. Genetic defects causing familial hypercholesterolaemia: identification of deletions and duplications in the LDL-receptor gene and summary of all mutations found in patients attending the Hammersmith Hospital Lipid Clinic. Atherosclerosis 2007;194:102-111

10. Taylor A, Tabrah S, Wang D, et al. Multiplex ARMS analysis to detect 13 common mutations in familial hypercholesterolaemia. Clin Genet 2007;71:561-568.

11. Alonso R, Defesche JC, Tejedor D, et al. Genetic diagnosis of familial hypercholesterolemia using a DNA-array based platform. Clin Biochem 2009:42:899-903.

12. Flicek P, Amode MR, Barrell D, et al. Ensembl 2012. Nucleic Acids Res 2012:40(database issue):D84-D90

13. Li H, Durbin R. Fast and accurate short read alignment with Burrows-Wheeler transform. Bioinformatics 2009;25:1754-1760.

14. McKenna A, Hanna M, Banks E, et al. The Genome Analysis Toolkit: a MapReduce framework for analyzing next-generation DNA sequencing data. Genome Res 2010;20:1297-1303.

15. Usifo $E$, Leigh $S E$, Whittall RA, et al. Low-density lipoprotein receptor gene familial hypercholesterolemia variant database: update and pathological assessment. Ann Hum Genet 2012;76:387-401.

16. The International HapMap Project. Nature 2003;426(6968):789-796.

17. Abecasis $G R$, Altshuler $D$, Auton $A$, et al. A map of human genome variation from population-scale sequencing. Nature 2010;467(7319):1061-1073.

18. Plagnol V, Curtis J, Epstein $M$, et al. A robust model for read count data in exome sequencing experiments and implications for copy number variant calling. Bioinformatics 2012;28:2747-2754.

19. Taylor A, Martin B, Wang D, Patel K, Humphries SE, Norbury G. Multiplex ligation-dependent probe amplification analysis to screen for deletions and duplications of the LDLR gene in patients with familial hypercholesterolaemia. Clin Genet 2009;76:69-75.

20. Walsh T, Lee MK, Casadei S, et al. Detection of inherited mutations for breast and ovarian cancer using genomic capture and massively parallel sequencing. Proc Natl Acad Sci USA 2010;107:12629-12633.

21. Redin C, Le Gras S, Mhamdi O, et al. Targeted high-throughput sequencing for diagnosis of genetically heterogeneous diseases: efficient mutation detection in Bardet-Biedl and Alström syndromes. J Med Genet 2012;49:502-512.

22. Goossens D, Moens LN, Nelis E, et al. Simultaneous mutation and copy number variation (CNV) detection by multiplex PCR-based GS-FLX sequencing. Hum Mutat 2009;30:472-476.

23. Ware JS, John S, Roberts AM, et al. Next generation diagnostics in inherited arrhythmia syndromes: a comparison of two approaches. J Cardiovasc Trans/ Res 2013;6:94-103

24. Hollants S, Redeker EJ, Matthijs G. Microfluidic amplification as a tool for massive parallel sequencing of the familial hypercholesterolemia genes. Clin Chem 2012;58:717-724.

25. Loman NJ, Misra RV, Dallman TJ, et al. Performance comparison of benchtop high-throughput sequencing platforms. Nat Biotechno/ 2012;30: 434-439. 
26. Gilles A, Meglécz E, Pech N, Ferreira S, Malausa T, Martin JF. Accuracy and quality assessment of $454 \mathrm{GS}-\mathrm{FLX}$ Titanium pyrosequencing. BMC Genomics 2011;12:245.

27. Dhawan P, Chang R, Mehta KD. Identification of essential nucleotides of the FP1 element responsible for enhancement of low density lipoprotein receptor gene transcription. Nucleic Acids Res 1997;25:4132-4138.

28. Chen WJ, Goldstein JL, Brown MS. NPXY, a sequence often found in cytoplasmic tails, is required for coated pit-mediated internalization of the low density lipoprotein receptor. J Bio/ Chem 1990;265:3116-3123.

29. Jensen HK, Jensen LG, Hansen PS, Faergeman O, Gregersen N. Two point mutations $(313+1 \mathrm{G} \rightarrow \mathrm{A}$ and $313+1 \mathrm{G} \rightarrow \mathrm{T})$ in the splice donor site of intron 3 of the low-density lipoprotein receptor gene are associated with familial hypercholesterolemia. Hum Mutat 1996;7:269-271.

30. Holla $\varnothing \mathrm{L}$, Nakken $S$, Mattingsdal $M$, et al. Effects of intronic mutations in the LDLR gene on pre-mRNA splicing: comparison of wet-lab and bioinformatics analyses. Mol Genet Metab 2009;96:245-252.
31. Motazacker MM, Pirruccello J, Huijgen R, et al. Advances in genetics show the need for extending screening strategies for autosomal dominant hypercholesterolaemia. Eur Heart J 2012;33:1360-1366.

32. Link E, Parish S, Armitage J, et al. SLCO1B1 variants and statininduced myopathy--a genomewide study. N Engl J Med 2008;359(8): 789-799.

33. Wilke RA, Ramsey LB, Johnson SG, et al.; Clinical Pharmacogenomics Implementation Consortium (CPIC). The clinical pharmacogenomics implementation consortium: CPIC guideline for SLCO1B1 and simvastatininduced myopathy. Clin Pharmacol Ther 2012;92:112-117.

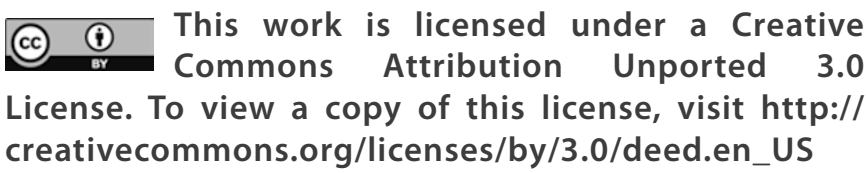

